the past. Whether there is any beneficial effect relative to the aging process and extension of the lifespan as observed in rats by Knoll ${ }^{19}$ remains to be substantiated in additional rats as well as in humans.

\section{Conclusions}

The assumption that the delayed need for levodopa therapy in early Parkinson's disease is due to some protective action of deprenyl rather than symptomatic therapy should remain hypothetical until some of these as yet unknown considerations have been fully validated.

With the knowledge of this complex and controversial background a fundamental question arises. Should physicians and their patients be sprinkling deprenyl on cornflakes? Much of this overenthusiasm is media and patient driven. It would appear that at this stage the evidence and indicators that may suggest a possible protective role for deprenyl in early Parkinson's disease, as well as in other neurodegenerative disorders, unfortunately remains dubious and as yet unestablished.

\section{REFERENCES}

1. Birkmayer W, Riederer P, Youdim MBH, et al. The potentiation of the anti-kinetic effect after L-Dopa treatment by an inhibitor of MAO-B, deprenyl. J Neural Transm 1975; 36: 303-326.

2. Lees AJ, Kohout LS, Shaw KM, et al. Deprenyl in Parkinson's disease. Lancet 1977; ii: 791-795.

3. Golbe LI, Lieberman AN, Muenter MD, et al. Deprenyl in the treatment of symptom fluctuations in advanced Parkinson's disease. Clin Neuropharmacol 1988; 11: 45-55.

4. Elizan TS, Yahr MD, Moros DA. Selegiline as an adjunct to conventional levodopa therapy in Parkinson's disease. Arch Neurol 1989; 46: 1280-1283.

5. Birkmayer W, Knoll J, Riederer P, et al. Increased life expectancy resulting from addition of L-deprenyl to madopar treatment in
Parkinson's disease: A long-term study. J Neural Transm 1985; 64: 113-27.

6. Csanda E, Tarczy M. Selegiline in early and late phases of Parkinson's disease. J Neural Transm 1987; Suppl 25: 105-114.

7. Elizan TS, Yahr MD, Moros DA. Selegiline use to prevent progression of Parkinson's disease. Arch Neurol 1989; 46: 1275-1279.

8. Myllyla VV, Sotaniemi KA, Tuominen J, et al. Selegiline as primary treatment in early phase Parkinson's disease. Acta Neurol Scand 1989; 126: 177-182.

9. Lieberman A, Thompson R, Fazzini E, et al. Deprenyl: Long term experience. Abstract, Neurology 1990; 40 (Suppl 1): 153.

10. Tetrud JW, Langston JW. The effect of deprenyl (selegiline) on the natural history of Parkinson's disease. Science 1989; 245: 519-522.

11. Parkinson Study Group. Effect of deprenyl on the progression of disability in early Parkinson's disease. N Engl J Med 1989; 321 : 1364-1371.

12. Kofman OS. Is deprenyl symptomatic or protective in Parkinson's disease? Parkinson Network 1990; Bull. 54: 1-2.

13. Kofman OS. Is deprenyl symptomatic or protective in Parkinson's disease? Author's Comments, Parkinson Network 1990; Bull. 55: 2.

14. Kofman OS. Antioxidative experimental therapies. Shoulson I, ed. Discussion, First International Congress of Movement Disorders. Washington, D.C. Apr. 25, 1990.

15. Kofman OS. Deprenyl. the protective vs. symptomatic effect. The national conference on Parkinson's disease. Victoria, B.C., September 8, 1990.

16. Landau WM. Clinical neuromythology IX - pyramid sale in the bucket shop: Datatop Bottoms Out. Neurology 1990; 40: 1337-40.

17. Sonsalla PK, Golbe LI. Deprenyl as prophylaxis against Parkinson's disease? Clin Neuropharmacol 1988; 11:500-511.

18. Markham CH, Diamond SG. Evidence to support early levodopa therapy in Parkinson's disease. Neurology 1981; 31: 125-31.

19. Knoll J. Extension of life span of rats by long-term deprenyl treatment. The Mount Sinai Journal of Medicine 1988; 55: 67-74.

From the Department of Medicine (Neurology). University of Toronto. Toronto

Reprint requests to: Dr. O.S. Kofman, 99 Avenue Road. Suite 608, Toronto, Ontario, Canada MSR 2G5

\title{
Deprenyl: The Exciting Possibility of Protective Effect
}

\author{
J. David Grimes
}

Despite the great benefits of levodopa therapy Parkinson's disease remains slowly progressive. The most exciting development in the pharmacotherapy of Parkinson's disease in the last few years has been the possibility that the progression of the disease may be related to exogenous or endogenous neuronal toxicity and that this may be improved with antioxidative therapy. 1,2

$A$ recent study involving 800 patients showed that the use of deprenyl ( $10 \mathrm{mg}$ per day) delays the onset of disability associated with early, otherwise untreated Parkinson's disease. In this doubleblind, placebo controlled study, the risk of having to start levodopa therapy (the end point of the study) was reduced by $57 \%$ for patients who received deprenyl. ${ }^{3}$ The question has been raised as to whether this delay in requirement for levodopa treatment is secondary to slowing of disease progression or mild symptom improvement. This controversy has resulted in the publication of inaccurate, biased, misinterpretations of available data. ${ }^{4}$
Deprenyl has been used as monotherapy for de novo Parkinson's disease in a number of studies. The majority of these studies have involved small numbers (20 to 56) of patients with variable study design. ${ }^{5-9}$ Csanda and Tarczy, ${ }^{5}$ showed that 20 of 30 patients treated with deprenyl monotherapy required other antiparkinsonian therapy within six months. Another study of 22 patients attempted to assess whether deprenyl halted the progression of the disease; it did not; and the study ended with the conclusion that it may still reduce the rate of progression. 6 The study of Myllyla concluded that deprenyl monotherapy has some efficacy but the study is complicated by the fact that anticholinergic drugs were allowed as adjuvant therapy. ${ }^{7}$ Terravainen has had experience that is probably closest to that of most clinical neurologists. ${ }^{8}$ In a study of 20 levodopa naive patients he concluded that deprenyl improved clinical neurological disability by about $10 \%$ compared to placebo. He felt that the difference was 
neither statistically nor clinically significant and that the results were compatible with the possibility that treatment with deprenyl alone does not significantly increase brain dopamine concentration. The interpretation of this study is complicated by the fact that the dose of deprenyl was increased up to $30 \mathrm{mg}$ daily. A study of 54 patients, which was similar in design to Datatop, including wash-in and wash-out assessments concluded that the significant delay in reaching end point was not due to a transient deprenyl therapeutic effect. ${ }^{9}$

The Datatop Study clearly shows that deprenyl has mild symptomatic benefit. ${ }^{3}$ The mean level of improvement was only about $10 \%$ when compared to baseline and it seems unlikely that this degree of improvement would account for the delay in requirement for levodopa therapy. It has been stated that deprenyl does not elicit an acute increase in dopaminergic activity. 10 Some trials with other drugs have shown a greater degree of improvement in placebo treated patients. "The weakness of the argument that the results of the Datatop Study are solely related to a symptomatic effect of deprenyl is demonstrated by the following. Some placebo and deprenyl treated patients showed symptomatic early improvement; some did not. End point was reached later in the patients who did not have early symptomatic improvement with deprenyl when compared to those placebo treated patients who had early benefit. This result remains strong and statistically significant. ${ }^{3}$

It has been suggested that studies of possible protective therapies for Parkinson's disease would be better if they included a comparison to the effects of standard antiparkinsonian agents. Large multi-centre trials are expensive, the potency of all the available antiparkinsonian agents is quite well known. Anticholinergics will improve the clinical features of Parkinson's disease by 10 to $25 \%$; amantadine is slightly more potent and benefits about two-thirds of patients. ${ }^{12}$ The efficacy of bromocriptine and levodopa in untreated patients is much better and is dose related. It seems an impossible task to design studies that would attempt to include any or all of these variables plus deprenyl as protective or symptomatic therapy. In addition, clinical experience also indicates that there is much less symptom worsening when deprenyl is stopped in levodopa untreated patients than on withdrawal of an anticholinergic, amantadine or low dose bromocriptine.

It has been shown that the clinical effect of deprenyl in levodopa treated patients was not related to amphetamine metabolites. ${ }^{13}$ Deprenyl has also been shown not to have a statistically significant antidepressant effect after three weeks of treatment at doses of $10 \mathrm{mg}$ daily. 14 This point is significant when one notes that the mild symptomatic improvement reported in the Datatop Study occurred in the first month.

There are some other reasons for the early treatment of Parkinson's disease patients with deprenyl. The dose of levo- dopa may be reduced about $30 \%$ and deprenyl treated patients have a lower incidence of the development of severe "on-off" fluctuations. ${ }^{5}$

Therefore, until a large single study involving 800 or more patients shows negative conclusions, or the final result of the Datatop Study reverses this initial optimism, I believe that mildly disabled Parkinson's disease patients should be treated with deprenyl. There seems reasonable hope that the course of their disease will be ameliorated to at least some degree. The next generation of protective therapy studies will involve other neurochemical manipulations with more selective, potent, agents. These are optimistic heady times for patients with neurodegenerative diseases and for those who care for them. Pessimism, and paralysis by analysis, will impede rather than facilitate, the development of new and more beneficial treatment regimes.

\section{REFERENCES}

1. Parkinson Study Group. Datatop: A multicenter controlled clinical trial in early Parkinson's disease. Arch Neurol 1989; 46: 1052 . 1060.

2. Grimes JD, Hassan MN, Thakar J. Antioxidant therapy in Parkinson's disease. Can J Neurol Sci 1987; 14: 483-487.

3. Parkinson Study Group. Effect of deprenyl on the progression of disability in early Parkinson's disease. N Engl J Med 1989; 321 : 1364-1371.

4. Landau WM. Pyramid sale in the bucket shop: Datatop bottoms out. Neurology 1990; 40: 1337-1339.

5. Csanda E, Tarczy M. Selegiline in the early late phases of Parkinson's disease. J Neural Transm 1987; 25: 105-113.

6. Elizan TS, Yahr MD, Moros DA, et al. Selegiline use to prevent progression of Parkinson's disease. Arch Neurol 1989; 46: 1275-1279.

7. Myllyla VV, Sotaniemi KA, Tuominen J, et al. Selegiline as primary treatment in early phase Parkinson's disease - an interim report. Acta Neurol Scand 1989; 126: 177-182.

8. Teravainen H. Selegiline in Parkinson's disease. Acta Neurol Scand 1990; 81: 333-336.

9. Tetrud JW, Langston JW. The effect of deprenyl (selegiline) on the natural history of Parkinson's disease. Science 1989; 245: 519-522.

10. Knoll J. Deprenyl (selegiline): the history of the its development and pharmacological action. Acta Neurol Scand 1983; 95: 57-80.

11. Diamond SG, Markham CH, Treciokas LJ. Double-blind trial of pergolide in Parkinson's disease. Neurology 1985; 35: 291-295.

12. Quinn NP. Anti-parkinsonian drugs today. Drugs 1984; $28: 236-262$.

13. Stern GM, Lees AJ, Hardie RJ, et al. Clinical and pharmacological problems of deprenyl (selegiline) treatment in Parkinson's disease. Acta Neurol Scand 1983; 95: 113-116.

14. Mann JJ, Fox-Aarons S, Wilner PJ, et al. A controlled study of antidepressant efficacy and side effects of (-) -Deprenyl. Arch Gen Psychiatry 1989; 46: 45-50.

From the Division of Neurology and Loeb Medical Research Institute, Ottawa Civic Hospital, University of Ottawa, Ottawa

Reprint requests to: Dr. J.D. Grimes, Division of Neurology and Loeb Medical Research Institute, Otrawa Civic Hospital, University of Ottawa, 1053 Carling Avenue, Ottawa. Ontario. Canada KIY $4 E 9$ 